Aim of the study: Important signalling pathways play fundamental roles in the pathogenesis of thyroid carcinoma (TC). PTEN, mTOR, PI3K-p85 and K-Ras are the principal factors involved in these signalling pathways. To immunohistochemically examine the expressions of PI3K, mTOR and PTEN in patients suffering from follicular TC, papillary TC or variants thereof, as well as to investigate KRAS mutations via PCR to determine their clinical and prognostic relevance to differentiated thyroid cancer.

Material and methods: The expression of PTEN, PI3K-p85 and mTOR was immunohistochemically examined, and the mutation of K-Ras was examined via PCR. The results obtained were compared to the clinico-pathologic characteristics of the patients.

Results: A significant correlation was found between p85 expression and lymphovascular invasions and between PTEN expression and multifocality $(p=0.048$ and $p=0.04$, respectively), and a correlation between p85 and capsular invasion was found, with a borderline statistical significance $(p=0.056)$. No expression of PTEN, p85 or Mtor was detected in normal tissue. K-Ras mutation was examined in 66 of the 101 patients (57.4\%), and the percentage of patients exhibiting a K-Ras mutation was $17.4 \%$. All of the patients exhibiting a K-Ras mutation were women $(p=0.047)$. The disease-free survival was 44.6 months (95\% Cl: 37.9-51.3) and was statistically significantly higher in the group that displayed level 1 or lower expression of $\mathrm{p} 85(p=0.043)$.

Conclusions: The expression levels of the aforementioned markers were significantly higher in TC cells than in normal tissue. A significant correlation was detected between K-Ras mutation and gender. This study demonstrates that p85 and PTEN are markers that should be evaluated in further studies of TC.

Key words: thyroid cancer, PTEN, mTOR, PI3K, K-Ras.

Contemp Oncol (Pozn) 2014; 18 (4): 234-240 DOI: $10.5114 /$ wo. 2014.43803

\section{Evaluation of PTEN, PI3K, MTOR, and KRAS expression and their clinical and prognostic relevance to differentiated thyroid carcinoma}

\author{
Berna B. Duman ${ }^{1}$, Oğuz I. Kara' ${ }^{1}$ Aysum Uğuz ${ }^{2}$, Berna T. Ates ${ }^{2}$
}

${ }^{1}$ Department of Medical Oncology, Medical Faculty, Cukurova University, Adana, Turkey 2Department of Pathology, Medical Faculty, Cukurova University, Adana, Turkey

\section{Introduction}

In thyroid carcinoma (TC), the phosphoinositide- $3(\mathrm{OH})$ kinase $(\mathrm{PI} 3 \mathrm{~K})$ / Akt and mitogen-activated protein (MAP) kinase pathways are significantly involved in tumourigenesis. After the activation of RTKs by growth factors, RET/PTC and RAS play a role in both pathways. PI3K, the mammalian target of rapamycin (mTOR), and Kirsten rat sarcoma (KRAS) signalling are responsible for cell growth, proliferation and survival. Phosphatase and tensin homolog (PTEN) is a tumour suppressor [1].

PI3Ks contain several subunits. Class I PI3Ks are well defined and consist of a p85 regulatory and a p110 catalytic subunit [2-5]. The PI3K/Akt pathway is activated as a result of the binding of the $\mathrm{p} 85$ subunit of $\mathrm{PI} 3 \mathrm{~K}$ to the subunits of activated tyrosine residues present on an activated growth factor receptor or via interaction with a RAS protein [6]. PTEN plays many roles in several subcellular locations, including that of a negative regulatory lipid protein phosphatase involved in the PI3K-associated signalling pathway. The primary function of PTEN is the negative regulation of the PI3K pathway, which it performs by removing the phosphate from position 3 of phosphoinositides via its phosphatase activity [7]. PTEN deactivation was detected in different types of carcinoma [8-10]. mTOR is a regulatory protein, involved in the PI3K/Akt/mTOR pathway, which increases the uptake of iodine from thyroid cells to promote cell proliferation and survival. Genetic alterations occurring in this pathway play a role in the progression of TC. mTOR is a highly attractive target via several therapeutic strategies [11]. RAS is an oncogene family that regulates two important signalling pathways in thyroid cancer: the MAP kinase/extracellular signal-regulated kinase (Ras/Raf/MEK/ERK) and PI3K/Akt signalling pathways. N-, H- and K-Ras are the three essential members of this family. The activation of oncogenic RAS causes point mutations that affect the GTP-binding domain (codon 12 or 13) in exon 1 or the GTPase domain (codon 61) in exon 2. These mutations fix the RAS proteins in an activated state, thereby causing chronic stimulation, genomic instability, additional mutations and malignant transformation of these genes. RAS mutations were detected in follicular adenomas and in the follicular variant of papillary thyroid carcinoma (PTC) $[12,13]$. In TC, the prevalence of RAS mutations is approximately $20-40 \%$. The role of RAS in the progression of thyroid tumours is unclear [1].

The important role that these genetic alterations play in TC is an important guide for the development of new gene-based diagnostic, prognostic and therapeutic strategies. Although the standard therapeutic strategy for the treatment of thyroid cancer still includes surgery, radioactive iodine (RAI) treatment and thyroid suppression therapy, an understanding of the specific factors that constitute the signalling pathways involved in TC has provided 
opportunities for targeted therapies [1, 14, 15]. As a result of the establishment of these targets of thyroid cancer, several targeted therapies, such as sorafenib, gefitinib, axitinib, motesanib, sunitinib, imatinib, and pazopanib, were generated. Clinical studies are ongoing to determine the effectiveness of these drugs in patients suffering from locally advanced and metastatic disease, who have exhibited resistance to previous therapeutic strategies (i.e. surgery and RAI) [14].

In light of these results, the aim of our study was to immunohistochemically examine the expressions of PI3K, mTOR and PTEN in patients suffering from follicular (FTC), papillary TC (PTC) or variants thereof, as well as to investigate KRAS mutations via PCR to determine their clinical and prognostic relevance to differentiated thyroid cancer.

\section{Material and methods}

\section{Patient selection}

This study enrolled a cohort of 101 patients suffering from differentiated thyroid carcinoma, who were diagnosed and followed between 2005 and 2010 by the Cukurova University Faculty of Medicine in Adana, Turkey. The patient database of the hospital was screened. The patients were staged based on the TNM staging system. The patients' demographic data, such as age, sex, city of residence, telephone number, date of diagnosis, concomitant systemic diseases, the presence of secondary cancer, family history of cancer, personal history of radiotherapy, tumour diameter, status of the lymph nodes, the presence of distant metastasis, the surgical method performed, therapies administered and characteristics including lymph vascular invasion (LVI), capsular invasion (CI) and multifocality of the existing pathological specimens, were recorded. Paraffin blocks of the tumour were retrieved from the pathology archives. All patients with inadequate follow-up data and those with inadequate pathology specimens were excluded from the study. A total of 160 patients with histologically confirmed differentiated thyroid cancer were selected for analysis in this study. Of these, paraffin embedded tissue material/tissue blocks were available from 133 patients, of which 106 were suitable for analysis. There was not adequate material from five patients, who were therefore excluded from the study. We evaluated a total of 101 cases of differentiated thyroid carcinoma. The mean age of the patients was $46.3 \pm 12.1$ years. Tissue specimens from a total of 101 patients suffering from DTC, normal thyroid tissue near the tumour specimens and 23 pathology specimens from patients suffering from Hashimoto thyroiditis (HT) were analysed. The paraffin blocks were stained for immunohistochemical analysis, and DNA samples were isolated for PCR analysis. This study was approved by the Cukurova University Faculty of Medicine Ethics Committee (approval date 02.12.2011, approval number 17).

\section{Performance of PCR of K-RAS}

First, DNA was isolated using a QIAmp DNA FFPE kit. The quantity of DNA was measured using a Nan photometer (Version: 7122VI.6.1, Serial number: 1562), and based on this result, real-time PCR of K-Ras was performed. For this purpose, an Entrogen kit specific for genomic mutations in K-Ras codon 12, 13 or 61 (Instrument Type: Applied Biosystems 7500 Real-Time PCR System) was used.

\section{Immunohistochemical staining for PTEN, PI3K and $\mathrm{mTOR}$}

Formalin-fixed, paraffin-embedded (FFPE) blocks of thyroid tumour specimens were retrieved and reviewed to confirm the presence of normal TC and normal thyroid tissue near the cancerous area or HT. Thyroidectomy material was used. The samples and controls were sectioned (5 micrometres) and stained using the Dako Autostainer Plus Staining System (Dako USA, Carpinteria, California (CA), USA). The primary antibodies used were: a PI3K-p85 monoclonal antibody (Clone SP62, M3624, Spring Bioscience, Pleasanton, CA, USA), a PTEN mouse monoclonal antibody (Clone 28H6, NCL-PTEN, Novocastra Laboratories, United Kingdom) and an mTOR rabbit anti-human polyclonal antibody (Clone N/A, Spring Bioscience, Pleasanton, CA, USA).

Finally, after rinsing with deionised water, the slides were counterstained using haematoxylin, dehydrated, mounted using a toluene-based mounting medium (Thermo Scientific Richard-Allan) and coverslipped. The samples were evaluated by two independent pathologists from the Cukurova University Department of Pathology. All of the samples were evaluated by the same investigators. The samples stained with PTEN, mTOR or p85 antibody were examined using a light microscope at a magnification of 400×. Positive staining was considered as nuclear staining for PTEN, cytoplasmic and membranous staining for mTOR and cytoplasmic staining for p85. The expressions of PTEN, mTOR and p85 in the tumour cells were graded between 0 and 3. No staining in the tumour cells was considered as 0 , while $1-10 \%, 10-50 \%$ and $>50 \%$ staining were defined as 1, 2 and 3, respectively (Figs. 1-3).

\section{Statistical analysis}

Statistical analysis of the data was performed using the SPSS 18.0 software package. The results were evaluated by an investigator from the Department of Biostatistics. The

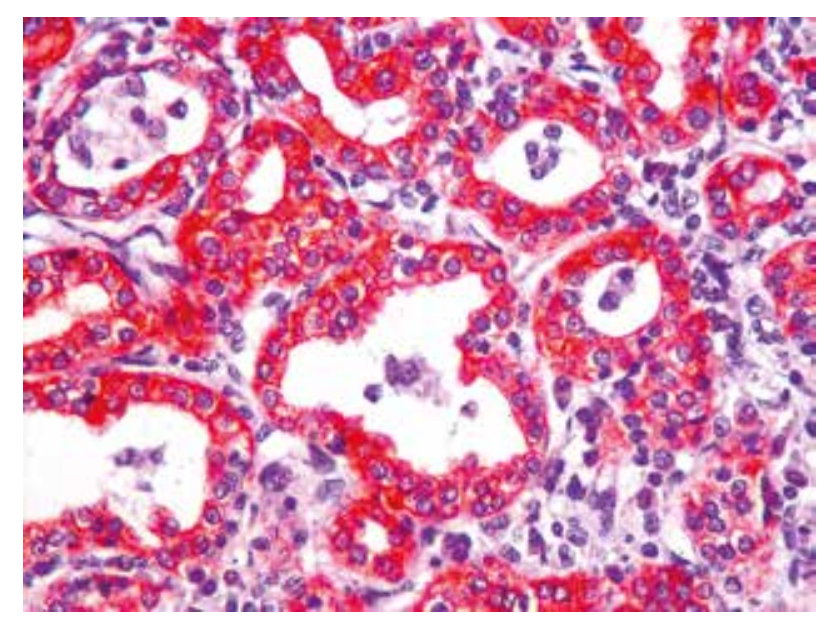

Fig. 1. $m$ TOR +3 positive staining $400 \times$ high field power 


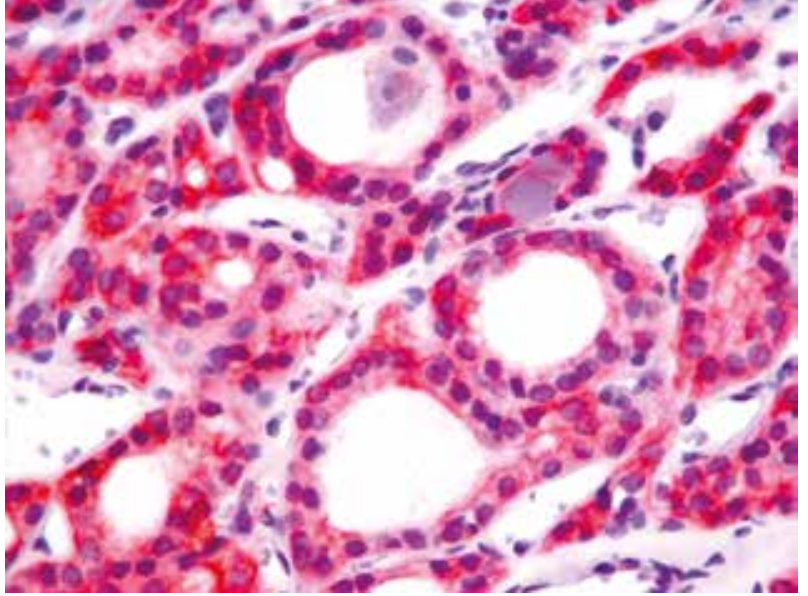

Fig. 2. p85 +3 positive staining $400 \times$ high field power

Table 1. Demographic and pathological characteristics of the patients

$\begin{array}{lcc} & \begin{array}{c}\text { Papillary carcinoma } \\ 81.2 \%(n=82)\end{array} & \begin{array}{c}\text { Follicular carcinoma } \\ 18.8 \%(n=19)\end{array} \\ \text { Gender } & \begin{array}{c}\text { Male: } 72.7 \%(n=16) \\ \text { Female: } 83.5 \%(n=66)\end{array} & \begin{array}{c}\text { Male: } 27.3 \%(n=6) \\ \text { Female: } 16.5 \%(n=13)\end{array} \\ \begin{array}{l}\text { Mean age } \\ \text { (age } \pm \text { SD })\end{array} & 45.32 \pm 12.7 & 45.74 \pm 12.04 \\ \text { LVI } & 13(68.4 \%) & \\ \begin{array}{l}\text { Capsule } \\ \text { invasion }\end{array} & 12(54.5 \%) & 10(31.6 \%) \\ \text { Multifocality } & 24(88.9 \%) & 3(11.1 \%) \\ \begin{array}{l}\text { Hashimato } \\ \text { Thyroiditis }\end{array} & 20(87 \%) & 3(13 \%) \\ \text { Stage } & \text { I: } 56(68.3 \%) & \text { I: } 11(55 \%) \\ & \text { II: } 19(23.2 \%) & \text { II: } 6(30 \%) \\ \text { III: } 3(4.9 \%) & \text { III: } 1(5 \%) \\ \text { IV: } 2(3.7 \%) & \text { IV: } 3(10 \%)\end{array}$

Table 2. Expression rates of PTEN, $m$ TOR and $p 85$

$\begin{array}{lccc}\text { Expression rate } & \text { PTEN; } n(\%) & \text { mTOR; } \mathrm{n}(\%) & \text { P85; } \mathrm{n}(\%) \\ 0 / 1+ & 30(29.7) & 3(3) & 26(25.7) \\ 2+ & 25(24.8) & 22(21.8) & 30(29.7) \\ 3+ & 46(45.5) & 76(75.2) & 45(44.6)\end{array}$

categorical measurements were expressed as the number and the percentage, while the numerical measurements were expressed as the mean and the standard deviation. Categorical measurements were compared between the groups using the $\chi^{2}$ test. Disease-free survival and PTEN, mTOR and PI3K-p85 expression were examined using the Log-Rank test with Kaplan-Meier analysis.

\section{Results}

This study included 101 patients. Of these patients, $81.2 \%(n=82)$ suffered from PTC and $18.8 \%(n=19)$ suffered from FTC. The mean age of the patients (mean age \pm SD) was $46.3 \pm 12.1$ years. Of these 101 patients, $78.2 \%$ $(n=79)$ were women and $21.8 \%(n=22)$ were men. The demographic and pathologic characteristics of the

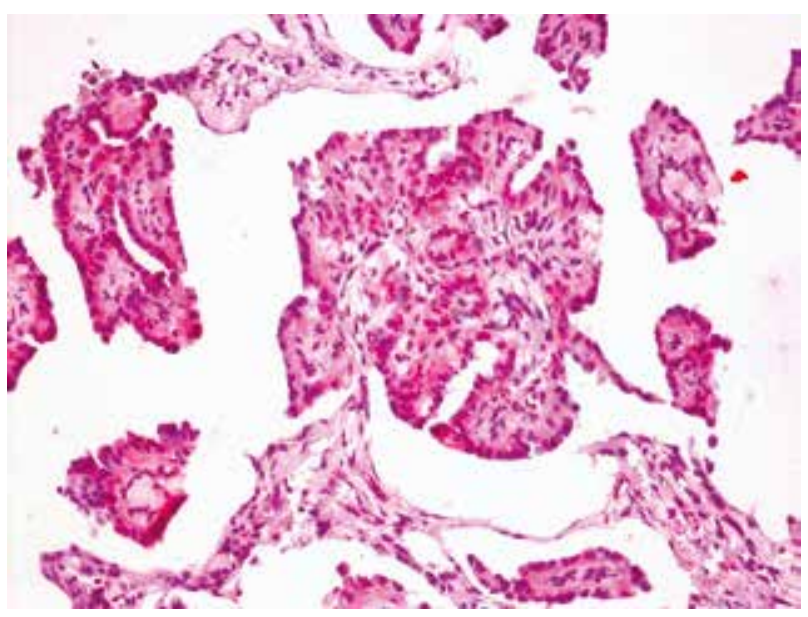

Fig. 3. PTEN +1 positive staining $400 \times$ high field power NOTE: The preparations stained with PTEN, mTOR and p85 antibodies were examined using a light microscope at a magnification of 400x. Positive staining was considered as nuclear staining for PTEN, cytoplasmic and membranous staining for mTOR and cytoplasmic staining for p85. PTEN, mTOR and p85 expressions of the tumour cells were graded between 0 and 3 . No staining in tumour cells was considered as 0 , whereas $1-10 \%, 10-50 \%$ and $>50 \%$ stainings were evaluated as negative, $+1,+2$ and +3 , respectively

patients are presented in Table 1. Of the patients, 89.1\% $(n=90)$ had undergone total thyroidectomy and $10.9 \%$ $(n=11)$ had undergone partial thyroidectomy. Neck dissection was performed on $10.9 \%(n=8)$ of the patients. In $5 \%$ of the patients $(n=5)$, lymph node involvement was detected. RAl treatment was administered to all patients except one, and only one patient received sorafenib treatment.

The normal thyroid tissue (areas near the tumour tissue lacking cancer cells) of all 101 patients was assessed for all of the biomarkers, and no expression was detected in the normal tissue. Twenty-three patients who suffered from HT were also analysed, and no staining for PTEN, PI3K-p85 or mTOR was detected. The rates of expression of PTEN, mTOR and $\mathrm{p} 85$ are presented in Table 2.

The tumour size (mean diameter $(\mathrm{cm}) \pm$ SD) and the expression of PTEN, mTOR and p85 were compared. No statistically significant association was detected between these groups $(p=0.439, p=0.818$ and $p=0.584$, respectively).

There was no significant correlation between $\mathrm{LVI}$ or $\mathrm{Cl}$ and PTEN expression, whereas a significant correlation was found between multimodality and PTEN expression. Level 3 PTEN expression was more frequent in patients suffering from thyroid cancer with multifocality $(p=0.04)$ (Table 3 ). No statistically significant correlation was found between mTOR expression and LVI, CI or multifocality $(p=0.392$, $p=0.65$ and $p=0.156$, respectively). Although there was no statistically significant correlation between multifocality and p85 expression, a statistically significant correlation was found between $\mathrm{LVI}$ and $\mathrm{p} 85$. The patients that expressed high levels of $p 85$ exhibited less LVI $(p=0.048)$, i.e. there was an inverse correlation. Of the 22 patients exhibiting $\mathrm{Cl}, 31.8 \%(n=7)$ had a score of 1 or lower, $45.5 \%$ $(n=10)$ had a score of 2 , and $22.7 \%(n=5)$ had a score of 3 , 
Table 3. Comparison of PTEN, mTOR and $\mathrm{p} 85$ by pathological characteristics

\begin{tabular}{|c|c|c|c|c|c|c|c|c|c|c|}
\hline \multicolumn{2}{|c|}{ Expression rate } & \multicolumn{2}{|c|}{ LVI, $n$ (\%) } & \multirow[t]{2}{*}{$p$} & \multicolumn{2}{|c|}{ Multifocality, $n$ (\%) } & \multirow[t]{2}{*}{$p$} & \multicolumn{2}{|c|}{$\mathrm{Cl}, n(\%)$} & \multirow[t]{2}{*}{$p$} \\
\hline & & + & - & & + & - & & + & - & \\
\hline PTEN & $\begin{array}{l}0 / 1+ \\
2+ \\
3+\end{array}$ & $\begin{array}{l}5(26.3) \\
7(36.8) \\
7(36.8)\end{array}$ & $\begin{array}{c}25(30.5) \\
18(22) \\
39(46.7)\end{array}$ & 0.39 & $\begin{array}{c}9(33.3) \\
2(7.4) \\
16(59.3)\end{array}$ & $\begin{array}{l}21(28.4) \\
23(31.1) \\
30(45.5)\end{array}$ & 0.04 & $\begin{array}{l}6(27.3) \\
9(40.9) \\
7(31.8)\end{array}$ & $\begin{array}{l}24(30.4) \\
16(20.3) \\
39(49.4)\end{array}$ & 0.778 \\
\hline mTOR & $\begin{array}{l}0 / 1+ \\
2+ \\
3+\end{array}$ & $\begin{array}{c}0 \\
6(31.6) \\
13(68.4)\end{array}$ & $\begin{array}{c}3(3.7) \\
16(19.5) \\
63(76.8)\end{array}$ & 0.39 & $\begin{array}{c}0 \\
9(33.3) \\
18(66.7)\end{array}$ & $\begin{array}{c}3(4.7) \\
13(17.5) \\
58(78.4)\end{array}$ & 0.15 & $\begin{array}{c}0 \\
5(22.7) \\
17(77.3)\end{array}$ & $\begin{array}{c}3(3.8) \\
17(21.5) \\
59(77.3)\end{array}$ & 0.65 \\
\hline P85 & $\begin{array}{l}0 / 1+ \\
2+ \\
3+\end{array}$ & $\begin{array}{c}4(21.1) \\
10(52.6) \\
5(26.3)\end{array}$ & $\begin{array}{l}22(26.8) \\
20(24.4) \\
40(48.4)\end{array}$ & 0.04 & $\begin{array}{l}10(38.5) \\
17(22.7) \\
17(22.7)\end{array}$ & $\begin{array}{l}16(21.6) \\
23(31.1) \\
35(47.3)\end{array}$ & 0.29 & $\begin{array}{c}7(31.8) \\
10(45.5) \\
5(22.7)\end{array}$ & $\begin{array}{l}19(24.1) \\
20(25.3) \\
40(50.6)\end{array}$ & 0.056 \\
\hline
\end{tabular}

for p85. Of the 79 patients lacking $\mathrm{Cl}, 50.6 \%(n=40)$ had a score of 3 for p85 expression $(p=0.056)$ (Table 3$)$.

During follow-up, recurrence was detected in only three patients. Of these three patients experiencing recurrence, two (66.7\%) exhibited local recurrence and one (33.3\%) exhibited distant metastasis. The expressions of PTEN, mTOR and $p 85$ and the rate of disease-free survival (DFS) were evaluated, but because all of the patients remained alive, the overall survival data could not be provided. In these three patients who experienced recurrence, no statistically significant correlation was observed for PTEN, mTOR or p85 staining ( $p=0.889, p=0.758$ and $p=0.76$, respectively) (Figs. 4-6). Recurrence was not found in any of the patients in whom K-ras mutation was analysed. DFS analysis was performed based on the expression levels of PTEN, mTOR and p85.

K-ras mutation: In $34.7 \%(n=35)$ of the 101 patients, K-ras mutation was impossible to analyse due to technical issues. Although $57.4 \%$ of the patients $(n=58)$ lacked any mutation, $7 \%(n=8)$ exhibited a mutation. Among the

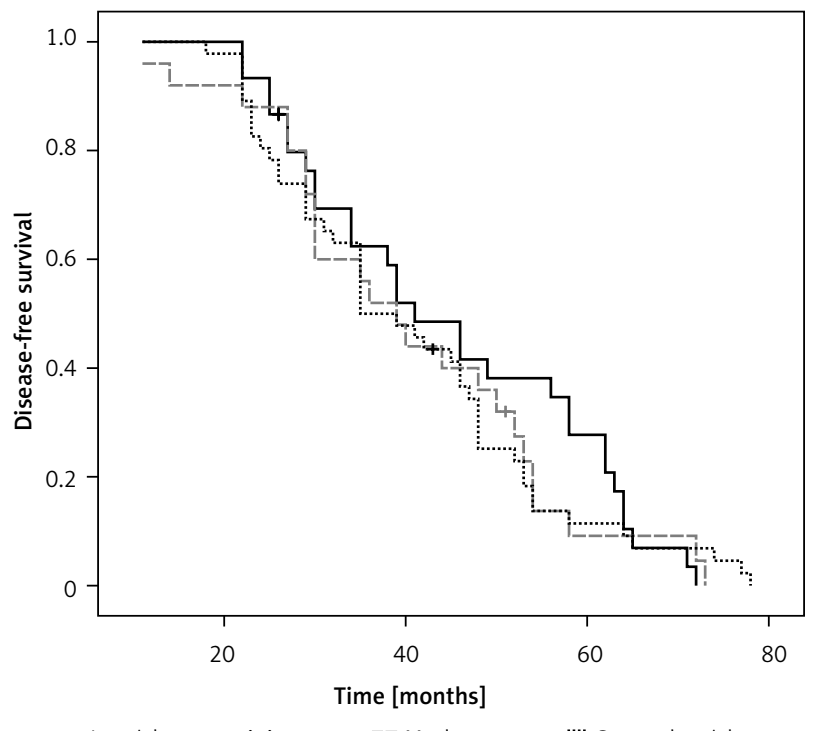

Fig. 4. PTEN expression and disease-free survival

Disease-free survival was found to be 44.8 months (95\% Cl: 38.9-50.7) in patients with PTEN expression $1(+) /(-), 40.6$ months $(95 \% \mathrm{Cl}: 34.1-$ $47.1)$ in patients with PTEN expression $2(+)$ and 40 months $(95 \% \mathrm{Cl}$ : $36.08-45.2)$ in patients with PTEN expression $3(+)(p=0.779)$ patients in whom a mutation was detected, six patients $(5.9 \%)$ exhibited a mutation in the $12^{\text {th }}$ codon of exon 1 , one patient (1\%) exhibited a mutation in the $13^{\text {th }}$ codon of exon 1 and one patient (1\%) exhibited a mutation in the $61^{\text {st }} \mathrm{co}-$ don of exon 2. Of the patients who exhibited a K-ras mutation in the $12^{\text {th }}$ codon of exon 1 , one patient (1\%) displayed a substitution from glycine to alanine, three patients (3\%) displayed a substitution from glycine to valine, one patient (1\%) displayed a substitution from glycine to arginine and one patient (1\%) displayed a substitution from glycine to cysteine. When KRAS mutation was compared by sex, none of the male patients $(n=20)$ exhibited a mutation, whereas $17.4 \%$ of the female patients $(n=8)$ exhibited a mutation. There was a statistically significant difference between these groups ( $p=0.047)$. When evaluated based on histological subtypes, $84.9 \%$ of the patients suffering from PTC lacked any mutation $(n=45)$, whereas $15.1 \%(n=8)$ exhibited a mutation. All of the patients suffering from FTC $(n=13)$ lacked any mutation. No statistically significant difference was found between these groups $(p=0.154)$.

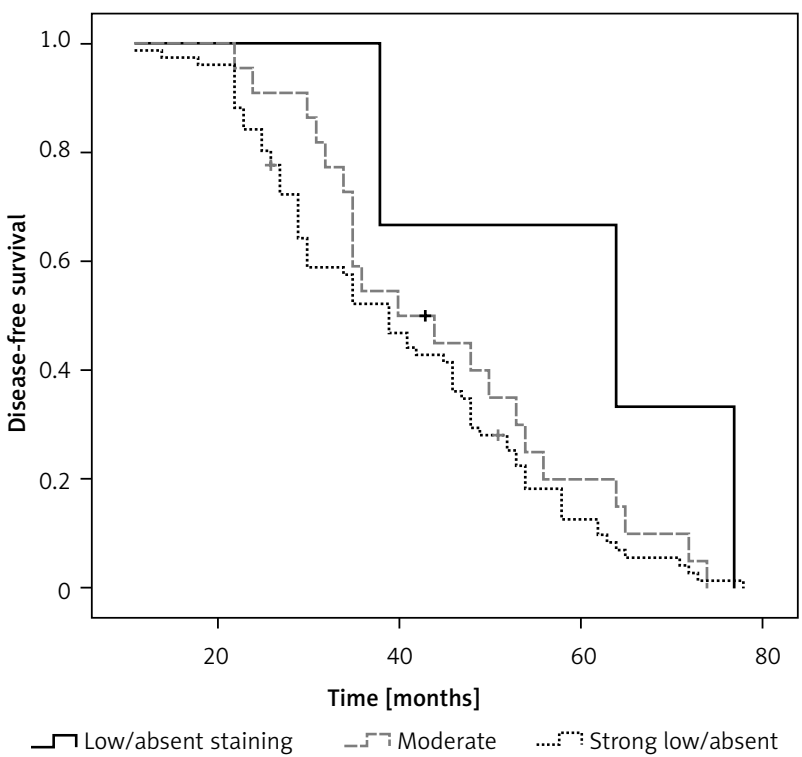

Fig. 5. mTOR expression and disease-free survival

Disease-free survival was found to be 59 months ( $95 \% \mathrm{Cl}$ : 37.1-82.1) in patients with mTOR expression $1(+) /(-), 45$ months $(95 \% \mathrm{Cl}: 38.5-$ 51.6) in patients with mTOR expression $2(+)$ and 40 months $(95 \% \mathrm{Cl}$ : $36.7-43.9)$ in patients with mTOR expression $3(+)(p=0.155)$ 


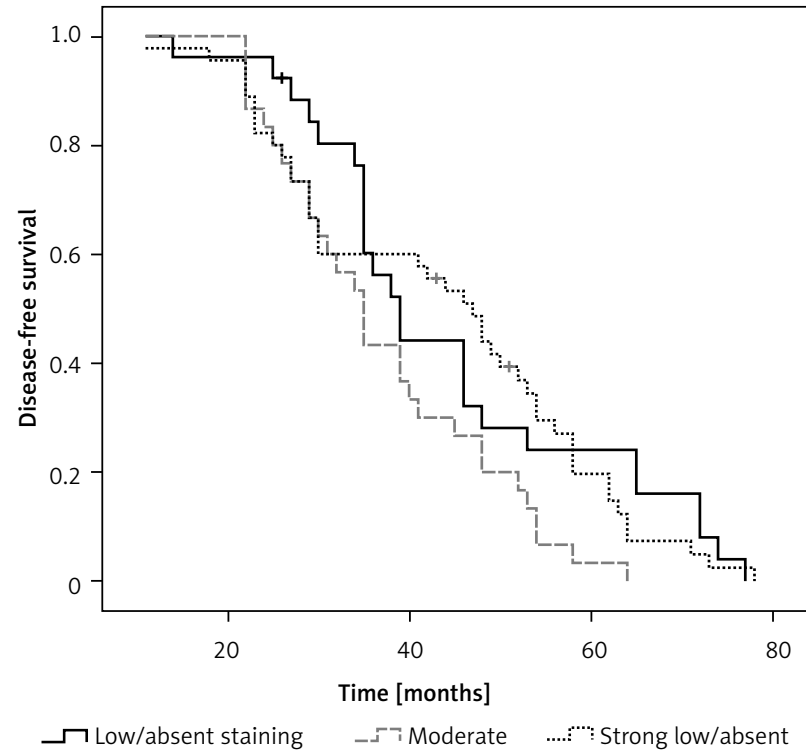

Fig. 6. p85 expression and disease-free survival

Median disease-free survival was found to be 44.6 months $(95 \% \mathrm{Cl}$ : $37.9-51.3)$ in patients with p85 expression $1(+) /(-), 37.1$ months $(95 \%$ $\mathrm{Cl}$ : 32.8-41.5) in patients with p85 expression $2(+)$ and 43.5 months $(95 \% \mathrm{Cl}: 38.4-48.6)$ in patients with p85 expression $3(+)(p=0.043)$

KRAS mutations were evaluated based on tumour size, $\mathrm{LVI}, \mathrm{Cl}$ and multifocality. No statistically significant difference was found between these groups ( $p=0.707, p=$ $=0.520, p=0.317$ and $p=0.418$, respectively) (Table 4).

\section{Discussion}

The understanding of specific factors in the signalling pathways involved in TC provide opportunities for targeted therapies [1, 14-17] Previously, these signalling pathways involved in TC have frequently been immunohistochemically investigated in rat and cancer cell culture models. In a study conducted by Kemmochi et al. [18], phosphorylated inactivated PTEN and active Akt isoforms were immunohistochemically evaluated using capsulated invasive follicular cell-derived carcinomas in rats. The expression of inactive PTEN and activated Akt isoforms, elements of the PI3K pathway, was found to be higher in capsular carcinomas compared to parenchymal carcinomas [18]. In a study conducted by Sozopoulos et al., the mutational status and the expression of mediators of the PI3K/Akt pathway were examined in PTC both genetically and immunohistochemically. Although these genetic alterations primarily manifest as phosphorylation and activation of Akt, increased active phosphorylated (p)-Akt was not detected using immunohistochemical methods [19].

Cowden syndrome is important because of the demonstrated role of the PI3K/AKt signalling pathway in the development of FTC. In TCs, especially FTC and ATC, somatic PTEN mutations may occur $[16,20,21]$. In TC, loss of heterozygosity or gene deletion were frequently detected in the PTEN gene [22]. Bruni et al. [23] demonstrated that PTEN inactivation played a role in thyroid carcinogenesis and that PTEN suppressor activity was mediated by the cyclin-dependent kinase inhibitor p27 $7^{\mathrm{kip} 119}$. In our study, when we examined PTEN expression immunohistochemically for loss of PTEN from the tissue, such a result might be caused by various mutations likely to occur in this gene. We did not detect a significant difference in PTEN expression between patients suffering from PTC or FTC ( $p=0.159)$.

PI3K-p85, mTOR and PTEN, constituents of the PI3K pathway, are highly expressed in HT and do not exhibit any expression in normal thyroid cells. Larson et al. [24] reported the expression of components of the PI3K pathway after immunohistochemical evaluation of HT and PTC thyroid tissue and normal thyroid tissue. While p-Akt, Akt1 and Akt2, components of the PI3K pathway, were highly expressed in $\mathrm{HT}$ and $\mathrm{TC}$ cells, they did not detect any expression in normal follicular cells $[24,25]$. No expression in normal thyroid tissue or HT tissue was detected in this study.

These factors act in concert in the pathway of carcinogenesis, and a loss of PTEN causes Akt activation. It was demonstrated that in mice, the thyroid gland proliferation that developed due to the PTEN deficiency was associated with mTOR activity [4]. mTOR, which is activated by PI3K and causes the proliferation of various cells, is an important effector of this pathway [25]. In another study performed by Yeager et al. [26], although the loss of PTEN was found to cause continuous autonomous and homogenous growth of the hyperplasic gland and the follicular adenoma in thyroid follicular cells in mice, it was also revealed that chronic PI3K/Akt activation led to mTOR/S6K1 activation $[11,26]$.

LOSS of PTEN and activation of the PI3K/Akt pathway are also associated with tumour growth, aggressiveness and poor prognosis [27]. In our study, we examined the clinical and prognostic characteristics as well as the expression of these factors. In previous studies, including animal studies, Akt1 activation was demonstrated to cause tumour invasion in PTC and FTC. In animal studies, PI3K/ Akt activation was found to play an important role in the development of FTC-related metastases [28]. It is understood that in $\mathrm{TCS}, \mathrm{Cl}$ and distant metastasis are important

Table 4. Comparison of Kras mutation by pathological characteristics

\begin{tabular}{|c|c|c|c|c|}
\hline & & \multicolumn{2}{|c|}{ K-ras } & \multirow[t]{2}{*}{$\mathrm{p}$} \\
\hline & & wild & mutant & \\
\hline LVI & $\begin{array}{l}\text { yes } 21.2 \%(n=14) \\
\text { no } 78.8 \%(n=52)\end{array}$ & $\begin{array}{l}19.6 \%(n=13) \\
68.1 \%(n=45)\end{array}$ & $\begin{array}{c}1.6 \%(n=1) \\
10.7 \%(n=7)\end{array}$ & 0.52 \\
\hline Capsule invasion & $\begin{array}{l}\text { yes } 27.3 \%(n=18) \\
\text { no } 72.7 \%(n=48)\end{array}$ & $\begin{array}{l}25.7 \%(n=17) \\
61.8 \%(n=41)\end{array}$ & $\begin{array}{c}1.6 \%(n=1) \\
10.9 \%(n=7)\end{array}$ & 0.317 \\
\hline Multifocality & $\begin{array}{l}\text { yes } 72.7 \%(n=48) \\
\text { no } 27.3 \%(n=18)\end{array}$ & $\begin{array}{c}62 \%(n=41) \\
24.2 \%(n=16)\end{array}$ & $\begin{array}{c}10.7 \%(n=7) \\
3.1 \%(n=2)\end{array}$ & 0.418 \\
\hline
\end{tabular}


parameters of unresponsiveness to RAl therapy, disease progression and mortality. Multiple studies are being performed to investigate several factors that affect the function of Akt, particularly Akt1, in tumour invasion and metastasis [28, 29].

A statistically significant association was found between p85 expression and LVI in this study $(p=0.048)$. A borderline correlation was detected between $\mathrm{p} 85$ expression and $\mathrm{Cl}(p=0.056)$. A significant correlation was found between PTEN expression and multifocality $(p=0.04)$.

RAS activates both the MAPK and PI3K/Akt pathways. Ras mutation, which was most commonly detected in TC, includes a N-Ras mutation in codon 61, which has been observed in other studies. H-Ras and N-Ras mutations in codon 61 or codon 12/13 were more rarely detected [13, 16, 20, 21, 30]. However, there are also some studies that demonstrated that K-Ras mutation was the most commonly detected mutation in TC [31, 32]. In the study performed by Ricarte-Filho et al. [33], the most commonly detected mutated isoform in poorly differentiated TC (PDTC) was N-ras [33]. In thyroid cancer, its prevalence is approximately $20-40 \%$ [6]. In other studies, this rate varied. Based on several studies, the prevalence rate ranges from $0-50 \%$ in PTC and from $14-62 \%$ in FTC $[31,33]$. In the study performed by Ricarte-Filho et al., it was found that in PDTC, RAS mutations were more common compared to BRAF mutations ( $12 \%$ vs. $44 \%, p=0.002$ ) [33]. In our study, we determined the mutation rate of K-RAS to be $17.4 \%$.

In some studies, Ras mutations were commonly detected in the follicular variant of FTC and PTC, in which $\mathrm{PI} 3 \mathrm{~K} /$ Akt pathway serves as the main signalling pathway [34-36]. Especially for FTCs, the primary activator of PI3K/ Akt pathway is a Ras mutation. In some studies, a good correlation was demonstrated between Ras mutation and Akt phosphorylation in TC $[22,31]$. All of our patients who exhibited a K-Ras mutation suffered from PTC. None of the patients suffering from FTC exhibited any K-Ras mutation. These results highlight the role of mutations of K-ras, a member of the Ras subfamily, in the development of PTC.

Some studies demonstrated that RAS mutation was a poor prognostic factor in the course of TC and was associated with a more aggressive course [33, 37]. In the study performed by Rivera et al., TC patients exhibiting a Ras mutation were found to have a better prognosis, and the median survival duration of these patients was 6.6 years [38].

RAS mutation has been correlated with histological characteristics, including loss of differentiation in the tumour and clinico-pathologic parameters that indicate tumour aggressiveness, including tumour size and vascular invasion [33]. Garcia-Rostan et al. concluded that RAS mutations correlated with aggressive tumour behaviour and poor prognosis and that they could be used as prognostic determinants in their study [31].

In our study, a correlation was detected only between sex and Kras mutation. All of the mutations were detected in women $(p=0.047)$. Despite the demonstration of this correlation, which had not been previously reported, we believe that the inequality of our patient population with respect to sex, such that females comprised a $69.7 \%$ majority of the total patient population, affected this result.
Concomitance of the physiological manifestations of the RAS mutation and PTEN down-regulation has been demonstrated to cause TC in transgenic mouse models [37]. Therefore, in our study, we examined more than one parameter that was involved in the same signalling pathway, but we could not detect any correlation between these various parameters and the subtype, the pathological features or the clinical and prognostic characteristics of the tumour. We did not detect any association between KRAS mutation and tumour size, disease stage, histological subtype, $\mathrm{LVI}, \mathrm{Cl}$ or multifocality $(p=0.707, p=0.68$, $p=0.368, p=0.52, p=0.317$ and $p=0.418$, respectively).

Surgery remains the primary treatment modality for PTC. RAl and thyroid hormone suppression often complement the treatment plan. Although thyroid hormone suppression may decrease the incidence of disease recurrence and RAI may treat metastases, lymph node dissection (LND) is the typical therapeutic strategy for clinically evident cervical lymph node metastases $[39,40]$. Specific inhibitors of the PI3K/AKt signalling pathway develops against several target molecules involved in this pathway may be important therapeutics for the treatment of thyroid cancer (inhibition of Akt phosphorylation using perifosine or inhibition of mTOR inhibition using temsirolimus) [41]. A specific therapy for oncogenic Ras mutations in thyroid cancer is still lacking. It was found that, in animal models, combined treatment with MEK and PI3K/AKT/mTOR pathway inhibitors was more efficient than monotherapy [33]. In the clinical studies performed, farnesyl transferase inhibitors targeted mutant RAS, which could, in future, be considered as a therapeutic for the treatment of poor and undifferentiated TCs [31].

In conclusion, these results are important for two reasons. First, these biomarkers have prognostic and perhaps therapeutic importance. In particular, p85 was the most relevant biomarker of those examined in this study for predicting DFS. The other interesting result of this study is that a statistically significant difference in K-ras mutation was detected between men and women.

\section{The authors declare no conflict of interest.}

\section{References}

1. Xing M. Genetic alterations in the phosphatidylinositol-3 kinase/ Akt pathway in thyroid cancer. Thyroid 2010; 20: 697-706.

2. Mian C, Barollo S, Pennelli G, et al. Molecular characteristics in papillary thyroid cancers (PTCS) with no 131 l uptake. Clin Endocrinol (Oxf) 2008; 68: 108-16.

3. Vivanco I, Sawyers CL. The phosphatidylinositol 3-Kinase AKT pathway in human cancer. Nat Rev Cancer 2002; 2: 489-501.

4. Saji M, Ringel MD. The PI3K-Akt-mTOR pathway in initiation and progression of thyroid tumors. Mol Cell Endocrinol 2010; 321: 20-8.

5. Legakis I, Syrigos K. Recent advances in molecular diagnosis of thyroid cancer. J Thyroid Res 2011; 2011: 384213.

6. McCubrey JA, Steelman LS, Abrams SL, et al. Roles of the RAF/ MEK/ERK and PI3K/PTEN/AKT pathways in malignant transformation and drug resistance. Adv Enzyme Regul 2006; 46: 249-79.

7. Di Cristofano A, Pandolfi PP. The multiple roles of PTEN in tumor suppression. Cell 2000; 100: 387-90.

8. Pieczyńska B, Wojtylak S, Zawrocki A, Biernat W. Analysis of PTEN, estrogen receptor $\alpha$ and progesterone receptor expression in en- 
dometrial hyperplasia using tissue microarray. Pol J Pathol 2011; 62: 133-8.

9. Waniczek D, Snietura M, Pigłowski W, Rdes J, Kopeć A, Młynarczyk-Liszka J, Rudzki M, Hudyka K, Arendt J, Lange D. Analysis of PTEN expression in large intestine polyps and its relation to the recognized histopathological and clinical risk factors for cancer development in this location. Contemp Oncol (Pozn) 2012; 16: 310-5.

10. Waniczek D, Śnietura M, Młynarczyk-Liszka J, Pigłowski W, Kopeć A, Lange D, Rudzki M, Arendt J. PTEN expression profiles in colorectal adenocarcinoma and its precancerous lesions. Pol J Pathol 2013; 64: 15-20.

11. Souza EC, Ferreira AC, Carvalho DP. The mTOR protein as a target in thyroid cancer. Expert Opin Ther Targets 2011; 15: 1099-112.

12. Vasko V, Ferrand M, Di Cristofaro J, Carayon P, Henry JF, de Micco C. Specific pattern of RAS oncogene mutations in follicular thyroid tumors. J Clin Endocrinol Metab 2003; 88: 2745-52.

13. Zhu Z, Gandhi M, Nikiforova MN, et al. Molecular profile and clinical-pathologic features of the follicular variant of papillary thyroid carcinoma. An unusually high prevalence of ras mutations. Am J Clin Pathol 2003; 120: 71.

14. Kapiteijn E, Schneider TC, Morreau H, Gelderblom H, Nortier JW, Smit JW. New treatment modalities in advanced thyroid cancer. Ann Oncol 2012; 23: 10-8.

15. Gut P, Matysiak-Grześ M, Fischbach J, Klimowicz A, Gryczyńska M, Ruchała M. Lack of TSH stimulation in patients with differentiated thyroid cancer - possible causes. Contemp Oncol (Pozn) 2012; 16: 273-5.

16. Hou P, Liu D, Shan Y, et al. Genetic alterations and their relationship in the phosphatidylinositol 3-kinase/Akt pathway in thyroid cancer. Clin Cancer Res 2007; 13: 1161-70.

17. Santarpia L, Myers JN, Sherman SI, et al. Genetic alterations in the RAS/RAF/mitogen-activated protein kinase and phosphatidylinositol 3-kinase/Akt signaling pathways in the follicular variant of papillary thyroid carcinoma. Cancer 2010; 116: 2974-83.

18. Kemmochi S, Fujimoto H, Woo GH, et al. Involvement of PTEN/ Akt signaling in capsular invasive carcinomas developed in a rat two-stage thyroid carcinogenesis model after promotion with sulfadimethoxine. J Cancer Res Clin Oncol 2011; 137: 723-32.

19. Sozopoulos E, Litsiou H, Voutsinas G, et al. Mutational and immu nohistochemical study of the PI3K/Akt pathway in papillary thyroid carcinoma in Greece. Endocr Pathol 2010; 21: 90-100.

20. Liu Z, Hou P, Ji M, et al. Highly prevalent genetic alterations in receptor tyrosine kinases and phosphatidylinositol 3-kinase/akt and mitogen-activated protein kinase pathways in anaplastic and follicular thyroid cancers. J Clin Endocrinol Metab 2008; 93 3106-16.

21. Wang Y, Hou P, Yu H, et al. High prevalence and mutual exclusivity of genetic alterations in the phosphatidylinositol-3-kinase/ akt pathway in thyroid tumors. J Clin Endocrinol Metab 2007; 92 2387-90.

22. Dahia PL, Marsh DJ, Zheng Z, et al. Somatic deletions and mutations in the Cowden disease gene, PTEN, in sporadic thyroid tumors. Cancer Res 1997; 57: 4710-3.

23. Bruni P, Boccia A, Baldassarre G, et al. PTEN expression is reduced in a subset of sporadic thyroid carcinomas: evidence that PTEN growth suppressing activity in thyroid cancer cells mediated by p27kip1. Oncogene 2000; 19: 3146-55.

24. Larson SD, Jackson LN, Riall TS, Uchida T, Thomas RP, Oiu S, Evers BM. Increased incidence of well-differentiated thyroid cancer associated with Hashimoto thyroiditis and the role of the PI3k/Akt pathway. J Am Coll Surg 2007; 204: 764-55.

25. Yeager N, Brewer C, Cai KO, Xu XX, Di Cristofano A. Mammalian target of rapamycin is the key effector of phosphatidylinositol-3-OH-initiated proliferative signals in the thyroid follicular epithelium. Cancer Res 2008; 68: 444-9.

26. Guertin DA, Sabatini DM. Defining the role of mTOR in cancer. Cancer Cell 2007; 12: 9-22.

27. Sansal I, Sellers WR. The biology and clinical relevance of the PTEN tumor suppressor pathway. J Clin Oncol 2004; 22: 2954-63.
28. Kim CS, Vasko VV, Kato Y, et al. AKT activation promotes metastasis in a mouse model of follicular thyroid carcinoma. Endocrinology 2005; 146: 4456-63.

29. Vasko V, Saji M, Hardy E, et al. Akt activation and localisation correlate with tumour invasion and oncogene expression in thyroid cancer. J Med Genet 2004; 41: 161-70.

30. Cantara S, Capezzone M, Marchisotta S, et al. Impact of proto-oncogene mutation detection in cytological specimens from thyroid nodules improves the diagnostic accuracy of cytology. J Clin Endocrinol Metab 2010; 95: 1365-9.

31. Garcia-Rostan G, Zhao H, Camp RL, et al. Ras mutations are associated with aggressive tumor phenotypes and poor prognosis in thyroid cancer. J Clin Oncol 2003; 21: 3226-35.

32. Costa AM, Herrero A, Fresno MF, et al. BRAF mutation associated with other genetic events identifies a subset of aggressive papillary thyroid carcinoma. Clin Endocrinol (Oxf) 2008; 68: 618-34.

33. Ricarte-Filho JC, Ryder M, Chitale DA, et al. Mutational profile of ad vanced primary and metastatic radioactive iodine-refractory thyroid cancers reveals distinct pathogenetic roles for BRAF, PIK3CA, and AKT1. Cancer Res 2009; 69: 4885-93.

34. Xing M. BRAF mutation in papillary thyroid cancer: pathogenic role, molecular bases, and clinical implications. Endocr Rev 2007 28: 742-62.

35. Xing M. Prognostic utility of BRAF mutation in papillary thyroid cancer. Mol Cell Endocrinol 2010; 321: 86-93.

36. Abubaker J, Jehan Z, Bavi P, et al. Clinicopathological analysis of papillary thyroid cancer with PIK3CA alterations in a Middle Eastern population. J Clin Endocrinol Metab 2008; 93: 611-8.

37. Miller KA, Yeager N, Baker K, Liao XH, Refetoff S, Di Cristofano A. Oncogenic Kras requires simultaneous PI3K signaling to induce ERK activation and transform thyroid epithelial cells in vivo. Cancer Res 2009; 69: 3689-94.

38. Rivera M, Ghossein RA, Schoder H, Gomez D, Larson SM, Tuttle RM. Histopathologic characterization of radioactive iodine-refractory fluorodeoxyglucose-positron emission tomography-positive thyroid carcinoma. Cancer 2008; 113: 48-56

39. Kaczka K, Fendler W, Borowiec M, Młynarski W, Celnik A, Pomorski L. Lymph node metastases in papillary thyroid cancer detected by quantitative real-time polymerase chain reaction for thyroglobulin and cytokeratine-19. Pol J Pathol 2013; 64: 90-5.

40. Lan Shi, Haiping Song, Huiping Zhu, Dapeng Li, Ning Zhang. Pat tern, predictors and recurrence of cervical lymph node metastases in papillary thyroid cancer. Contemp Oncol (Pozn) 2013; 17: 504-9.

41. Liu D, Hou P, Liu Z, Wu G, Xing M. Genetic alterations in the phosphoinositide 3-kinase/Akt signaling pathway confer sensitivity of thyroid cancer cells to therapeutic targeting of Akt and mammalian target of rapamycin. Cancer Res 2009; 69: 7311-9.

\section{Address for correspondence}

Berna Bozkurt Duman MD

Department of Oncology

Medical Faculty, Cukurova University

01330 Adana, Turkey

e-mail: berboz@hotmail.com

Submitted: 18.09 .2013

Accepted: $\quad 3.02 .2014$ 